\title{
Exclusive Test and its Applications to Fault Diagnosis
}

\author{
Vishwani D. Agrawal* Dong Hyun Baik, Yong Chang Kim and Kewal K. Saluja \\ Agere Systems \\ 4 Connell Drive \\ Berkeley Heights, NJ 07922 \\ vishwani02@yahoo.com \\ University of Wisconsin-Madison \\ Department of Electrical and Computer Engineering \\ Madison, WI 53706 \\ \{dbaik,kimy,saluja\}@ece.wisc.edu
}

\begin{abstract}
We introduce a new type of test, called exclusive test, and discuss its application to fault diagnosis in combinational circuits. A test that detects exactly one fault from a given pair of faults is called an exclusive test. In general, generation of an exclusive test by a conventional automatic test generator requires a model of the circuit with multiple-faults. We describe an ATPG model that transforms the cxclusive tost problcm into a single-fault test generation problem. We present a generalized diagnostic method and illustrate the use of exclusive lests in improving lhe diagnostic resolution of a tcst sct. Results of diagnosis with cxclusive tcsts for ISCAS85 benchmark circuits are included.
\end{abstract}

\section{Introduction}

Most diagnostic procedures are based on single-fault tests. As a result, the "symptom" or "signature" produced by an actual defect often does not map onto the output generated by a single-fault simulator. Nevertheless, the single-fault dictionary approach $[5,6,15]$ is frequently used. The resolution of a diagnostic procedure can be improved if we could find tests for suspected fault set which is identified by the single-fault dictionary. We can also benefit from tests that "exclusively" detect one fault, but not the others, in the suspected fault set. Attempts were made to generate such test in $[9,14]$ using a conventional single fault model. Ilowever, those method require specialized ATPG or multiple test generations.

In this paper, we define exclusive test, present a method of generating it without any modification of ATPG and gives its application to diagnosis. In Section 2, we provide a model for exclusive test generation and fault simulation. An example is included to illustrate the use of exchusive test to improve the diagnostic resolution of a fault dictionary. In Section 3, we present a generalized algorithm for fault diagnosis using exclusive tests. To demonstrate how exclusive tests help improve the diagnostic resolution of a combinational circuit, we include diagnostic results of the proposed method for ISCAS85 circuits in Section 4.

${ }^{*}$ Visiting Professor, Dept. of ECE, Rutgers University, Piscataway, New Jersey.

\section{Exclusive Test}

An "Exclusive test" is an input vector that detects only one fault from a pair of targeted faults at a primary output [1]. The general exclusive test problem can be formulated for a pair of fault sets. For simplicity, however, we will study it for a pair of faults, $f_{1}$ and $f_{2}$. An exclusive test must detect exactly one of these faults. Consider a fault frec circuit $C_{0} . C_{1}$ and $C_{2}$ are the same circuit with faults $f_{1}$ and $f_{2}$, respectively. The circuit is assumed to be combinational and can have any number of inputs. For clarity, we will only consider single output functions. The Boolcan satisfiability formulation of the exclusive test problem is,

$$
\left(C_{0} \oplus C_{1}\right) \oplus\left(C_{0} \oplus C_{2}\right)=1
$$

which simplifies to,

$$
C_{1} \oplus C_{2}=1
$$

and may also be expressed as,

$$
\left(C_{0} \oplus C_{0}\right) \oplus\left(C_{1} \oplus C_{2}\right)=1
$$

Equation 2 is a condition for distinguishing between the two faults. The complementary condition, $C_{1} \oplus C_{2}=0$, is known as the indistinguishability condition [5], and when satisfied by all inputs makes the two faults equivalent. Equation 3 indicates that an exclusive test is a test for a double fault in two copies of the circuit under test producing a single output through an exclusive-OR gate, and each copy contains a different single fault. This ATPG model, shown in Figure 1, has been used to derive exclusive tests by a multi-valued logic systom [9]. We take an altcrnative approach to adapt the problem to a single fault ATPG. We make the following observations:

- If no exclusive test exists for two faults then either they are equivalent or both faults are redundant. Although not often recognizcd, all redundant faults of a combinational circuit form an equivalent fault set.

- If two faults are independent then any test that detects either one of them is an exclusive test for the pair. Two faults are defined as independent [2] if no vector can detect both of them. 


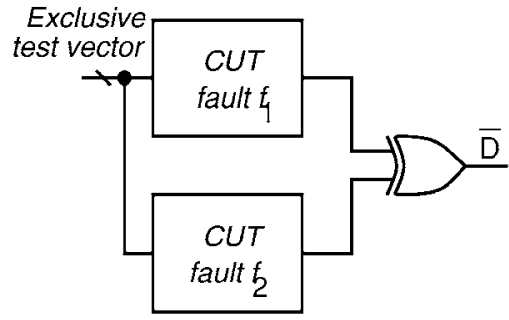

Figure 1: Generation of exclusive test.

Table 1: Fault dictionary enhancement by exclusive test.

\begin{tabular}{|c|c|c|c|}
\hline \multicolumn{2}{|c|}{$100 \%$ coverage tests } & \multicolumn{2}{|c|}{ Exclusive test added } \\
\hline $\begin{array}{c}\text { Test } \\
\text { syndrome }\end{array}$ & $\begin{array}{l}\text { Mapped } \\
\text { faults }\end{array}$ & $\begin{array}{c}\text { Test } \\
\text { syndrome }\end{array}$ & $\begin{array}{l}\text { Diag. } \\
\text { fault }\end{array}$ \\
\hline 0000001 & $c_{1}$ & 0000001000 & $c_{1}$ \\
\hline 0000011 & $b_{0}, \epsilon_{1}, i_{1}, k_{1}$ & 0000011000 & $b_{0}$ \\
\hline 0000100 & $n_{1}$ & 0000011100 & $e_{1}$ \\
\hline 0001000 & $b_{1}, h_{1}, m_{1}$ & 0000100000 & $n_{1}$ \\
\hline 0010000 & $l_{1}$ & 0001000000 & $b_{1}$ \\
\hline 0011000 & $i_{0}$ & 0001000001 & $m_{1}$ \\
\hline 0110100 & $a_{0}$ & 0001000010 & $h_{1}$ \\
\hline 0111100 & $z_{0}$ & 0010000010 & $l_{1}$ \\
\hline 1000000 & $d_{1}, g_{1}$ & 0011000011 & $i_{0}$ \\
\hline 1000011 & $a_{1}, z_{1}$ & 0110100010 & $a_{0}$ \\
\hline & & 0111100011 & $z_{0}$ \\
\hline & & 1000000000 & $\mathrm{~d}_{1}$ \\
\hline & & 1000011100 & $\mathbf{a}_{1}$ \\
\hline 0000000 & $\mathbf{f}_{1}, j_{1}$ & 0000000000 & $\overline{f_{1}}$ \\
\hline
\end{tabular}

\subsection{Application}

We illustrate the use of exclusive test in the generation of a diagnostic fault dictionary [5] through an example. Consider the circuit shown in Figure 2. Seven tests, $T_{1}=0000, T_{2}=1100, T_{3}=1000, T_{4}=0011, T_{5}=$ $1111, T_{6}=0111$ and $T_{7}=0101$, were generated by an ATPG program [7].

We obtain a total of 19 faults via structural equivalent fault collapsing, namely, $\left(a_{0}, a_{1}, b_{0}, b_{1}, c_{1}, d_{1}, e_{1}\right.$, $\left.f_{1}, g_{1}, h_{1}, i_{0}, i_{1}, j_{1}, k_{1}, l_{1}, m_{1}, n_{1}, z_{0}, z_{1}\right)$ where the subscripts denote the stuck-at values. The fault dictionary is given in Table 1. For the seven tests, each fault has a syndrome that is a seven-bit vector [5]. The $j t h$ bit $t_{j}$ of this vector is 1 if test $T_{j}$ detects that fault. Two faults, $f_{1}, j_{1}$, are redundant and are not detected by the seven tests. These redundant faults always have all-0 syndromes regardless of applied tests. The second column shows the mapped faults exhibiting the syndromes shown in the first column. Thus, the fault $b_{0}(b$ s-a- 0$)$, detected by $T_{6}$ and $T_{7}$, has a test syndrome 0000011 . Three other faults, $e_{1}, i_{1}$ and $k_{1}$, also have the same test syndrome as $b_{0}$ and those are not distinguishable by the given tests. Additional test(s) are required to resolve this ambiguity. This can be achieved by using exclusive tests for these faults. Figure 3 shows exclusive test generation for the fault pair $\left(b_{0}, e_{1}\right)$. This previously proposed model [10] allows detection of multiple faults by a single-fault ATPG [7], which generates the vector $T_{8}=0110$. The vector, which distinguishes between faults $b_{0}$ and $e_{1}$, is appended to the existing vector set

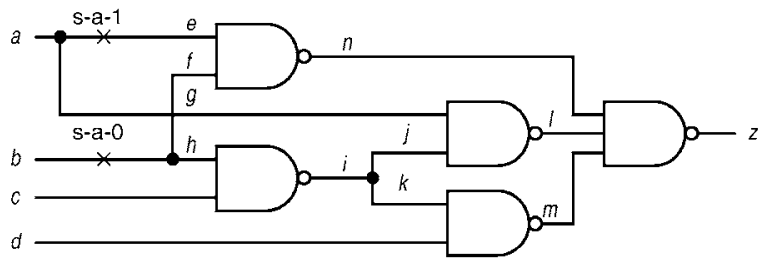

Figure 2: An example for diagnostic tests.

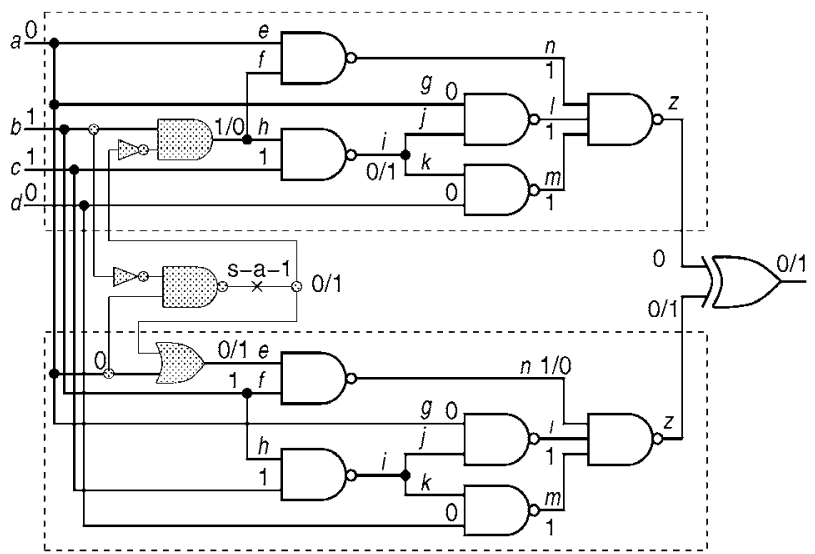

Figure 3: Exclusive test for the fault pair $\left(b_{0}, e_{1}\right)$.

and the fault dictionary is updated. Two additional tests, $T_{9}=1010$ and $T_{10}=1001$, which distinguish between faults of pairs $\left(b_{1}, h_{1}\right)$ and $\left(b_{1}, m_{1}\right)$, respectively, are also appended. However, there is no test for fault pairs $\left(a_{1}, z_{1}\right),\left(i_{1}, k_{1}\right),\left(b_{0}, i_{1}\right),\left(b_{0}, k_{1}\right)$ and $\left(d_{1}, g_{1}\right)$ indicating these pairs are equivalent pairs. These equivalent fault pairs form three equivalent fault classes $\left\{\mathbf{a}_{\mathbf{1}}, z_{1}\right\}$, $\left\{\mathbf{b}_{\mathbf{0}}, i_{1}, k_{1}\right\}$ and $\left\{\mathbf{d}_{\mathbf{1}}, g_{1}\right\}$. Since a fault in the equivalent fault class cannot be distinguished from other faults in the same equivalent class, we list only one fault (shown in boldface) to represent each equivalent class. The resulting test syndromes and diagnosed faults (Diag. fault) are shown in the last two columns of Table 1. Now every fault in the list can be diagnosed and the faults representing equivalent fault classes are shown in bold face. Only two fault pairs out of three pairs, $\left(i_{1}, k_{1}\right),\left(b_{0}, i_{1}\right),\left(b_{0}, k_{1}\right)$, are required to be tested to establish equivalence of all three faults. Also, it should be noted that redundant fault $f_{1}$ also represents redundant fault set which has all-0 syndrome.

\section{Fault Diagnosis}

Equivalent fault classes. Any given set of faults can be divided into subsets of equivalent faults. All equivalent faults produce identical circuit behavior at all primary outputs that are observed during test [5]. These fault subsets are called equivalent fault classes or simply equivalent classes. They have the following properties:

- A fault can belong to exactly one equivalent class.

- All redundant faults belong to the same equivalence class, which also includes the fault-free circuit or the "no fault" condition. 


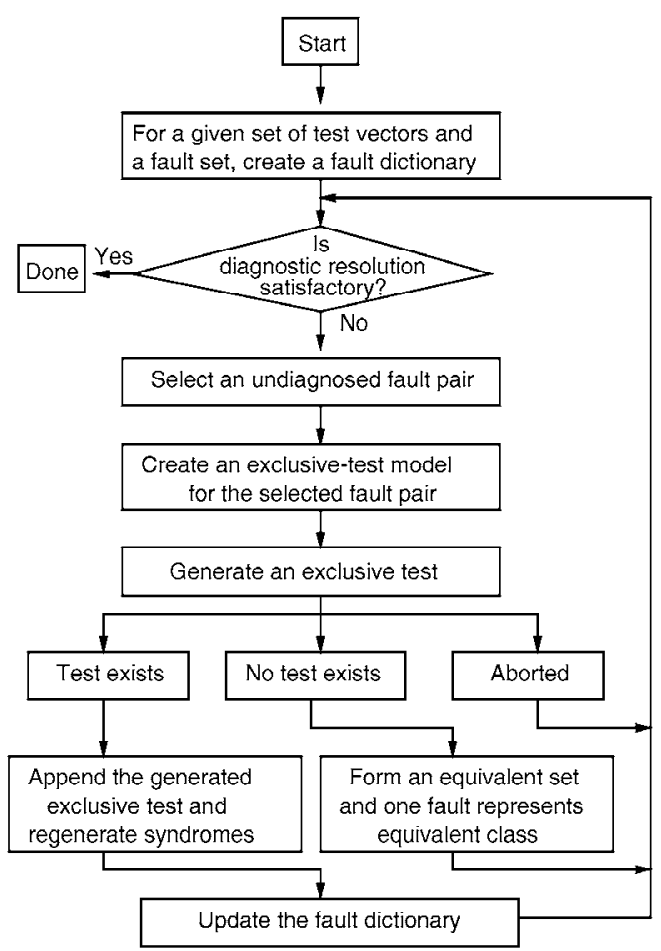

Figure 4: Diagnosis with exclusive tests.

- Faults in the same equivalence class cannot be distinguished from each other by any test set.

Diagnosis. A perfect diagnosis refers to the highest resolution, i.e., identification of a single suspect (or equivalence (lass). A diagnosis procedure may consider only onc fault from cach cquivalence class or all faults which belong to the equivalence class from the given fault set. The complete list of faults in the suspected equivalence class is more comprehensive and may be more useful for the purpose of repair.

Given a test set and a set of faults, each fault has a test syndrome or its decimal equivalent, diagnostic number [1]. We observe:

- A fault with a unique syndrome that differs from the syndrome of all other faults is classified as "uniquely diagnosed" or simply "diagnosed". A set of faults with the same syndrome forms an "undiagnosed fault set," which is further analyzed.

- Faults with all-0 syndrome are either redundant or not detectable by the given test set. However, the faults proven to be redundant fall into a diagnosed equivalent fault class. Otherwise, their redundant status is not known. This set of faults also forms an undiagnosed fault set and is analyzed further.

- Faults from an undiagnosed fault set can belong to either the same equivalence class (i.e., are indistinguishable) or different equivalent fault classes but not distinguished due to the inadequacy of the test set. Exclusive tests are generated for pairs of faults those have identical syndrome. This pair wise strategy is based on the property of fault equivalence, which is frequently used for finding the equivalence classes [13]. Suppose that we have an undiagnosed fault set with $k>1$ faults. We choose a pair of faults and attempt to generate an exclusive test. We have the following cases.

1. If an exclusive test is found for a pair of faults, the test is augmented and simulated to reclassify the fault set. As a result, an undiagnosed fault set now splits into two sets, each guaranteed to contain fewer then $k$ faults.

2. If no exclusive test exists, then the two faults belong to the same equivalence class. In this case, any one of this pair can represent the equivalent fault class and the number of faults in the set becomes $k-1$.

The number of faults in an undiagnosed set, $k$, is guaranteed to be reduced in both cases and undiagnosed set will eventually be fully diagnosed when $k$ becomes 1. However, exclusive test generation could be aborted due to backtrack limit or time constraint. In this particular case, the aborted fault pair will be excluded from further exclusive test generation.

We introduce an important measure called diagnostic resolution (DR) to quantify the quality of the diagnosis. T)R is defined as follow:

$$
D R=\frac{\text { number of faults }}{\text { number of syndromes }}
$$

D) $R$ gives an average number of faults per fault class (or syndrome). The equivalences of faults is unknown before the exclusive test generation. Thus we assume that all faults can be uniquely diagnosable and cach fault is counted once. During the diagnostic test generation, we reduce the number of faults as we discover more equivalent fault sets. A perfect DR of 1.0 is achieved if all cquivalont fault classes are identificd (cach equivalence class is represented by one fault).

For the example in Section 2.1, the initial DR is 1.55 since there are a total of 17 faulis and 11 different syndromes ( 7 diagnosed fault classes including redundant fault class and 4 undiagnosed fault sets). After the exclusive tests, the DR becomes 1.00; there are total of 14 faulis and 10 uniquely diagnosed faults and 4 diagnosed equivalent faults including redundant fault class.

Figure 4 shows a generalized diagnosis procedure using exclusive tests. For a given test set, we construct the fault dictionary using the definitions above. Depending on the test and the fault set, we may possibly have two different types of fault sets: diagnosed fault sets (may be uniquely diagnosed fault or diagnosed equivalent fault sets including the redundant fault set) and undiagnosed fault sets(two or more faults have same syndromes). If the diagnostic resolution is not satisfactory, we select a pair of faults to be diagnosed from an undiagnosed fault set. We generate an exclusive test model, generate a test and update the fault dictionary using the guidelines above. If the exclusive test exists, we simulate the generated test and regroup faults to 
Table 2: Diagnostic results : ISCAS85 circuits.

\begin{tabular}{|c|c|c|c|c|c|c|c|c|}
\hline Circuit names & $\mathrm{c} 17^{*}$ & $\mathrm{c} 432^{*}$ & $c 499^{*}$ & $c 880^{*}$ & $c 1355^{*}$ & $c 1908^{*}$ & $c 2670^{*}$ & $c 3540^{*}$ \\
\hline \multicolumn{9}{|c|}{ Detection tests } \\
\hline Number of detection tests & 6 & 82 & 58 & 104 & 104 & 176 & 236 & 239 \\
\hline Number of eq. collapsed faults & 22 & 524 & 758 & 942 & 1574 & 1879 & 2747 & 3428 \\
\hline Number of redundant faults & 0 & 4 & 0 & 5 & 0 & 4 & 84 & 90 \\
\hline Number of aborled faulds & 0 & 0 & 32 & 2 & 32 & 27 & 884 & 81 \\
\hline Number of detected faults & 22 & 520 & 726 & 935 & 1542 & 1848 & 1779 & 3257 \\
\hline Fault coverage $(\%)$ & 100.00 & 99.24 & 95.78 & 99.26 & 97.97 & 98.35 & 64.76 & 95.01 \\
\hline Fault efficiency $(\%)$ & 100.00 & 100.00 & 95.78 & 99.79 & 97.97 & 98.56 & 66.8 & 97.57 \\
\hline \multicolumn{9}{|c|}{ Diagnosis with detection tests } \\
\hline Number of faults & 22 & 520 & 726 & 935 & 1542 & 1848 & 1779 & 3257 \\
\hline Number of syndromes & 14 & 426 & 691 & 789 & 873 & 1450 & 1285 & 2706 \\
\hline Number of diagnosed faults & 9 & 354 & 661 & 686 & 360 & 1121 & 972 & 2351 \\
\hline Diagnostic resolution & 1.57 & 1.22 & 1.05 & 1.19 & 1.77 & 1.27 & 1.38 & 1.20 \\
\hline Maximum faulds per symdrome & 4 & 5 & 4 & 6 & 11 & 8 & 11 & 12 \\
\hline \multicolumn{9}{|c|}{ Diagnosis with detection and exclusive tests } \\
\hline Number of faults & 22 & 520 & 726 & 935 & 1542 & 1848 & 1779 & 3256 \\
\hline Number of syndromes & 22 & 506 & 710 & 870 & 902 & 1579 & 1385 & 2844 \\
\hline Number of diagnosed faulls & 22 & 492 & 694 & 808 & 366 & 1331 & 1097 & 2559 \\
\hline Diagnostic resolution & 1 & 1.03 & 1.02 & 1.07 & 1.71 & 1.17 & 1.28 & 1.14 \\
\hline Maximum faults per syndrome & 1 & 2 & 2 & 3 & 3 & 8 & 11 & 8 \\
\hline Total test vectors & 11 & 126 & 72 & 152 & 129 & 262 & 293 & 328 \\
\hline Number of exclusive tests & 5 & 44 & 14 & 48 & 25 & 86 & 57 & 89 \\
\hline Number of equivalent pairs found & 0 & 0 & 0 & 0 & 0 & 0 & 0 & 1 \\
\hline Number of aborted pairs & 0 & 14 & 16 & 79 & 744 & 321 & 630 & 662 \\
\hline
\end{tabular}

update fault dictionary. We repeat fault pair select, exclusive fault test generation, and fault dictionary update steps until a desired DR is obtained.

Major limitations of dictionary based diagnosis methods are that they require intensive computing and substantial storage space. However, our diagnosis methods can also be used for adaptive diagnosis as follows. First, obtain as much diagnosis information as possible using initial test set. During the actual diagnosis, we can start diagnosis using obtained information. If a fault is uniquely identified, we can terminate the diagnosis procedure. However, if the fault is found to be an element of undiagnosed fault set, then we can resolve this ambiguity by using our proposed method.

\section{Results}

We have presented a detailed flow chart (Figure 4) for the fault diagnosis procedure in Section 3. A typical diagnosis procedure starts with a test set (also called a detection test set or detection tests) and a list of faults. In general, only those faults detected by the detection tests or the subset of detected faults are identified and included for diagnosis. However, we excluded redundant faults and aborted faults from diagnosis due to the fact that a set of redundant faults are already di agnosed as one equivalent fault set, and aborted faults are generally aborted again for generation of exclusive test. Since no diagnostic method can distinguish between structurally equivalent faults, we identify struc turally equivalent faults and consider only one representative fault from each structurally equivalent fault sel. For these reasons, we slart with slructurally collapscd fault sct and consider only the detcetcd faults for diagnosis.

Since our exclusive test generation model is defined for a single output circuit, we converted ISC $\Lambda$ S 85 benchmark circuits to single output circuits by adding an "xor tree", a network of Exclusive-Or (xor) gates, to their outputs. A similar technique called "parity-scan design" was used to compact [8] thc outputs of scanregisters to minimize scan-in and scan-out effort. For the subsequent discussion, we use * to denote a modified circuil wilh a single oulpul xor lree al ils oulpuls.

It should be noted that an exclusive test for the modified single output circuit is also an exclusive test for the original multiple output circuit. However, the converse is not true since there may exist exclusive tests for multiple output circuits which are not the exclusive tests for single output circuit due to masking caused by xor-tiree.

For each modified ISCAS85 benchmark circuit, we use a combinational ATPG to generate fault detection tests for faults in the original multiple output circuit (faults in the xor tree are not considered) and all detected faults are to be diagnosed. We then proceed with the diagnosis phase using exclusive test method. Table 2 summarizes the results of our diagnosis and these are explained below.

First row lists the names of the ISC AS85 circuits used in our study for fault diagnosis. The remaining table is divided into three separate blocks. Each column contains the results for each modeled ISCAS 85 benchmark circuit and circuits. The first block, listed under the heading Detection tests, contains initial test generation results and the corresponding statistics. In this block the Number of detection tests is the number of test vectors generated by the combinational A'I'P(i that are to be used as the initial test set for diagnosis. The remaining six rows in this block summarize the statistics of this test set. Number of eq. collapsed faults is the number of faults obtained by structural equivalent col- 
lapsing, excluding faults in the xor tree. Number of redundant faults is the number of faults identified re dundant (untestable) in the modified circuits. In all cases, the backtrack limit was not set to be very high to mimic the realistic enviromment in which status of some faults remains aborted during the test gencration phase.

The second block in the Table 2, listed under the heading Diagnosis with detection tests, shows the results of initial fault dictionary construction using only the detection tests. Number of faults is the number of fanlts to be diagnosed, which is same as the Number of detected faults from the first block. Number of syndromes is the number of distinct syndromes obtained by the detection tests. Number of diagnosed faults is the number of faults which have unique syndromes, thus uniquely diagnosed by the detection tests. Diagnostic resolution gives an average number of faults per syndrome and Maximum faults per syndrome gives a maximum number of faults associated with a syndrome. These two parameters quantify the effectiveness of our diagnosis since DR indicates how well faults are distributcd among all syndromes and the Maximum faults per syndrome indicate the worse distribution among all syndromes. For the computation of DRs, we assume all detected fauls are uniquely diagnosable and each fault is countcd once since we have no information rcgarding fault equivalence at this stage of diagnosis.

'I'he third block, under the heading Inagnosis with detection and exclusive tests, shows the results and statistics of the fault dictionary after generation of exclusive tests. The first five rows have similar meaning to the second block, except for Number of diagnosed faults now contains diagnosed equivalent fault sets. As can be seen from the table, DRs and Maximum faults per syndrome are generally improved for most of the circuits. Total test vectors are obtained by adding the number of generated exclusive test vectors and the detection tests. The fault pairs that were found to be equivalent are listcd in the row Number of cquivalent pairs found. Since we did not change the default backtrack limit, the ATPG aborted many exclusive test generations and this value is listed under Number of aborted pairs. Only in onc case (circuit c3540) a fault pair was identificd to bc equivalent. This fact is reflected in the computation of diagnostic resolution.

\subsection{Fault Equivalences}

Traditional diagnosis methods $[3,11,12,16,17,18]$ rely only on the fault simulation to create fault dictionary. For this approach, the quality of diagnosis is limited by the supplied test sets. Often, some faults cannot be diagnosed by the supplied test set when there exist some other test sets that can diagnose those faults. Another limitation of simulation-only based diagnosis methods is that they are unable to prove if certain faults are equivalent (cannot be distinguished) with respect to all tests; it can only establish some faults are equivalent with respect to the given test set. In [4], Amyeen et al
Table 3: Diagnostic results for $\mathrm{C} 432$

\begin{tabular}{|c|r|r|r|}
\hline Circuit names & $c 432^{*}$ & $c 432$ & $c 432$ \\
\hline \hline \multicolumn{3}{|c|}{ Detection tests } \\
\hline Numbcr of detcction tests & 82 & 82 & 82 \\
Number of eq. collapsed faults & 524 & 524 & 524 \\
Number of redundant faults & 4 & 4 & 4 \\
Number of aborled faults & 0 & 0 & 0 \\
Number of detected faults & 520 & 520 & 520 \\
Fault coveragc(\%) & 99.24 & 99.24 & 99.24 \\
Fault efficiency(\%) & 100 & 100 & 100 \\
\hline \hline \multicolumn{3}{|c|}{ Diagnosis with detection tests } \\
\hline Number of faults & 520 & 520 & 520 \\
Numbcr of syndromes & 428 & 495 & 500 \\
Number of diagnosed faults & 354 & 471 & 479 \\
Diagnostic resolution & 1.22 & 1.05 & 1.04 \\
Maximum faults per syndronte & 5 & 5 & 4 \\
\hline \hline \multicolumn{3}{|c|}{ Diagnosis with detection and exclusive tests } \\
\hline Number of faults & 506 & 507 & 507 \\
Number of syndromes & 506 & 507 & 507 \\
Number of diagnosed faults & 492 & 494 & 494 \\
Diagnostic resolution & 1.00 & 1.00 & 1.00 \\
Maximum faults pcr syndrome & 1 & 1 & 1 \\
Total test vectors & 131 & 131 & 123 \\
Number of exclusive tests & 49 & 13 & 41 \\
Number uf equivalenl pair found & 14 & 13 & 13 \\
Number of aborted pair & 0 & 0 & 0 \\
\hline
\end{tabular}

expanded the method of identification of fault equivalence based on fault implication of the faulty values and functional evaluation at the dominator gate, where all the effects of the fault must be propagated through to reach outputs. This method offered an improved fault equivalence identification. However, it has two major limitations namely requiring a special tool and its inability to identify all equivalent fault classes. In [9, 14], similar approach was used to generate diagnostic test patterns and identify all fault equivalneces with their specialized A'I'PG. Our proposed method is different from previous methods since the proposed method can identify all faults equivalences using any conventional STPG without modification. However, we need to sub stantially increase the backtrack limit as well as the test generation time in order to determine fault equivalences for all undiagnosed faults. We set out an objective of perfect DR (=1) for the circuit c132*. The results of this study are given in Table 3 . All aborted fault pairs in Table 2 are now identified as 14 diagnosed equivalent fault sels and each has two faults per sel. Consequently, number of fault to be diagnosed is reduced to 506 since 14 out of 28 faults represent each equivalent fault class. Note that in this case the appended vectors are different than those generated in Table 2.

\subsection{Multiple Output Circuits}

In general, we do not have to modify a circuit to have a single output. Because the exclusive test generation model can be extended to a multiple output circuit by XORing each pair of outputs (outputs from the CUT with fault $f_{1}$ and the corresponding outputs from the CUT with fault $f_{2}$ in Figure 1). An exclusive test based diagnosis of a multiple output circuit is less restrictive than the circuit modified by an XOR tree to have a sin gle output as discussed in the previous sections. 'I'he 
multiple output model provides more outputs for propagating and observing the fault effects than the single output model. Further, if $f_{1}$ and $f_{2}$ are detected on two different outputs of the multiple output circuit, then $f_{1}$ and $f_{2}$ can be distinguished and diagnosed. Clearly, any test sct obtaincd using a singlc output model is guaranteed to diagnose all corresponding faults in the multiple output circuit. Similarly, if a pair of faults are found to be equivalent in the multiple output model, that pair of faults is guarantecd to be cquivalcnt in the single output model. We can use these relationships to find the appropriate diagnostic method for multiple outpul circuils. This can help reduce the number of vectors as well as equivalent fault classes.

The sccond data column of Tablc 3 shows the rcsults of applying the diagnostic test set generated for c432* (c432 modified by adding an xor tree) to diagnose unmodified c432. When the test sel (total of 131 vcctors) is applicd to c432, only 13 fault pairs (total 26 faults) needed exclusive test generation to establish their equivalences. Notice that one additional fault is distinguishable in c 432 than c $432^{*}$ since one of 14 equivalent pairs in single output model is uniquely diagnosed as two faults. The third data column of Table 3 shows the results of applying our proposed diagnosis method directly to 432 (without using the test set derived for single output modified circuit). The new diagnostic test set using the c432 resulted in same number of faults (507), syndromes (507), diagnosed faults and T)R (1.00) when compared to the results from second data column. The notable difference is a reduction in the size of test set (123 versus 131 vectors) due to increased observability through multiple outputs.

\section{Conclusion}

In this paper, we defined new type of test, exclusive test, and modeling method to allow conventional ATPG to generate exclusive test. We also presented a comprehensive diagnosis procedure using exclusive test to improve fault diagnosis. Our results for ISCAS85 benchmark circuits indicate that we can achicve very high diagnostic resolutions using our method.

Exclusive test based diagnosis method is different from the traditional approaches since we use both fault simulation and test generation for fault diagnosis. Our use of exclusive test guarantees that all faults can be diagnosed and all equivalent fault sets can be identified regardless of given test sets. With our exclusive test diagnosis method, we derive additional tests required for the diagnosis when the supplicd test set fails to diagnose all faults.

\section{References}

[1] V. D. Agrawal and K. K. Saluja, "Antitest, Exclusive Test, and Concurrent Test." Available from authors (URL http://www.ece.wisc.edu/ va), Dec. 2001.
[2] S. B. Akers, C. Juseph, and B. Krishmanurthy, "On the Role of Independent Fault Sets in the Generation of Minimal Test Sets," in Proc. International Test Conf., 1987, pp. 1100-1107.

[3] H. Al-Asaad and J. P. Hayes, "Logic Design Validation via Simulation and Automatic Test Pattern Generation," J. Electronic Testing: Theory and Applications, vol. 16, no. 6, pp. 575-589, Dec. 2000.

[1] M. E. Amyeen, W. K. Fuchs, I. Pomeranz, and V. Boppana, "Implication and Evaluation Techniques for Proving Fault Equivalence," in Proc. 17th IEEL VLSI Test Symp., 1999, pp. 201-207.

[5] M. L. Bushnell and V. D. Agrawal, Essentials of Electronic Testing for Digital, Memory and Mixed-Signal VLSI Circuits. Boston: Kluwer Academic Publisher, 2000.

[6] H. Y. Chang, E. G. Manning, and G. Metze, Fault Diagnosis of Digital Systems. New York: WileyInterscience, 1970.

[7] W.-T. Cheng and T. J. Chakraborty, "Gentest: An Automatic Test Generation System for Sequential Cir-

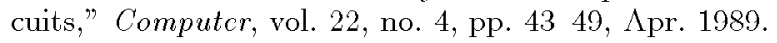

[8] H. Fujiwara and $\Lambda$. Yamamoto, "Parity-Scan Dcsign to Reduce the Cost of 'l'est Application," in Proc. Internalional Test Conf., 1992, pp. 283-292.

[9] T. Gruning, U. Mahulsted, and H. Koopmeiners, "DIATEST: A Fast Diagnostic Test Pattern Generator for Combinational Circuits," in International Conf. on Computer-Aided Design, 1991, pp. 194-197.

[10] Y. C. Kim, V. D. Agrawal, and K. K. Saluja, "Multiple Faults: Modeling, Simulation and Test," in Proc. 15th International Conf. VLSI Design, Jan. 2002.

「11) E. M.Rudnick, W. K. Fuchs, and J. H. Patel, "Diagnostic Fault Simulation of Sequential Circuits," in Proc. International Test Conf., Sept. 1992, pp. 178-186.

[12] I. Pomeranz and S. M. Reddy, "Location of Stuck-at. Faults and Bridging Faults Based on Circuit Partitioning," IEEE Trans. on Computers, vol. 47, pp. 11241135, Oct. 1998.

[13] A. V. S. S. Prasad, V. D. Agrawal, and M. V. Atre, " $\Lambda$ Ncw Algorithm for Global Fault Collapsing into Equivalence and Dominance Cells," in Proc. International Test Conf., Oct. 2002.

[14] J. Savir and J. Roth, "Testing for, and Distimguishing between Failures," in Proc. 12th Fault Tolerant Computing Symposium, 1982, pp. 165-172.

[15] W. R. Simpson and J. W. Sheppard, System Test and Diagnosis. Boston: Kluwer Academic Publishers, 1994.

[16] N. Sridhar and M. S. Hsiao, "On Efficient Error Diagnosis of I)igital (ircuits," in Pros. International 'lest. Conf., 2001, pp. 678-687.

[17] H. Takahashi, K. O. Boaleng, K. K. Saluja, and Y. Takamatsu, "On Diagnosing Multiple Stuck-at Faults Using Multiple and Single Fault Simulation in Combinational Circuits," IEEE Trans. on ComputerAided Design of Integrated Circuits and Systems, vol. 21, no. 3, pp. 362-368, March 2002.

[18] S. Venkataraman and S. B. Drummonds, "Poirot: Applications of a Logic Fault Diagnosis Tool," IEEE Design 8 Test of Computers, vol. 3, pp. 19-30, Jan. 2001. 